\title{
OPTIMIZACIÓN DEL COSTO DEL SISTEMA DE APROVECHAMIENTO DE MADERA EN BOSQUES NATURALES DE Pinus caribaea
}

\author{
Fidel Cándano Acosta ${ }^{1}$, Angelo Marcio Pinto Leite ${ }^{2}$, José Luis Martínez Cantón ${ }^{3}$
}

(recibido: 13 de julio de 2010; acepto: 28 de octubre de 2011)

\begin{abstract}
RESUMEN: El trabajo se realizó en bosques naturales de Pinus caribaea en áreas de la empresa forestal La Palma en la provincia de Pinar del Río - Cuba. El objetivo de la investigación fue minimizar el costo del sistema de aprovechamiento de madera a partir de la interacción entre el costo de camino y el costo de arrastre en base a la densidad de camino y patios de carga, además de la interacción del costo de camino y el costo del transporte de madera en base a la calidad de la capa de rodamiento del camino. Los resultados de la investigación muestran una reducción de la densidad de caminos y patios de carga, con la correspondiente disminución del costo de sistema de aprovechamiento de $0,45 \$ / \mathrm{m}^{3}$. Además se logró mejorar la calidad de la capa de rodamiento del camino sin alterar el costo total de construcción de este, propiciando una disminución de 1,01 $\$ / \mathrm{m}^{3}$ de madera transportada. En general, el costo del sistema de aprovechamiento se reduce en 1,46\$/ $\mathrm{m}^{3}$. También se hace una contribución considerable en la disminución del impacto al ecosistema al reducir $44 \%$ el área utilizada para la construcción de caminos y patios en relación al método tradicional.
\end{abstract}

Palabras-clave: Cosecha de madera, densidad de caminos, costo mínimo.

\section{COST OPTIMIZATION OF THE SYSTEM OF USE OF WOOD IN NATURAL FORESTS DE Pinus caribaea}

\begin{abstract}
The work was carried out in natural forests of Pinus caribaea in areas of the forest company "La Palma" in the province of Pinar del Río in Cuba. The objective of the investigation was to minimize the cost of harvesting by combining the costs of haulage and road and load patio densities. In addition, the study also takes into consideration the interaction of costs of transportation and the thickness and quality of the layer of gravel on the road. The results of the investigation have shown a significant increase in the spacing between roads and load patios with the decrease of US $\$ 0.45 / \mathrm{m}^{3}$. It was possible to improve the quality of the layer of gravel on the road without changing the construction costs significantly, which led to a cost reduction of US $\$ 1.01 / \mathrm{m}^{3}$. The overall cost considering the new system decreases in US\$1.46/ $\mathrm{m}^{3}$. Another significant contribution is made by decreasing the impact to the ecosystem once the reduction on road density may reach around $44 \%$ in comparison to the current density.
\end{abstract}

Key words: Harvesting, road density, minumum cost.

\section{INTRODUCCION}

El costo del sistema de cosecha de madera incide directamente sobre las ganancias de las empresas vinculadas al sector forestal. Desde la década del 40 del pasado siglo varios científicos han investigado los procedimientos para minimizar el costo de las operaciones de cosecha de madera (BECKER, 1994; LANFORD; STOKES, 1995; MALINOVSKI; MALINOVSKI, 2002; PETERS, 1978; SESSIONS, 1992; SESSIONS; GUANGDA, 1987; SESSIONS; HUAT, 1989; SUDDARTH; HEINRICH, 1964).

La eficiencia económica del sistema de aprovechamiento depende de varios factores tales como: condiciones geomorfológicas y climáticas de las áreas de tala, características de los árboles a aprovechar, tipo de maquinaria utilizada y las características de la mano de obra, como demuestran los resultados obtenidos por Amaral et al. (1998), Peters (1978), Sessions (1992) y Silva et al. (1991).

Para optimizar el costo del sistema de aprovechamiento de madera, se pueden utilizar diferentes vías; El diseño y construcción de nuevas máquinas y herramientas, la capacitación e incentivos de los obreros y demás trabajadores vinculados con el sistema de aprovechamiento y el perfeccionamiento de los métodos de trabajo en base a una planificación y ejecución más

\footnotetext{
${ }^{1}$ Ingeniero Forestal, Profesor Dr. en Ciencias Forestales - Departamento de Engenharia Florestal - Universidade do Estado de Mato Grosso/UNEMAT Rua F2, 203 - 78.580-000 - Alta Floresta, MT, Brasil - fidel.candano@yahoo.com.br

${ }^{2}$ Ingeniero Forestal, Profesor Dr. en Ciencias Forestales - Departamento de Engenharia Florestal - Universidade Federal dos Vales do Jequitinhonha e Mucuri/UFVJM - Rua da Glória, 187 - 39.100-000 - Diamantina, MG, Brasil - ampleite@ig.com.br

${ }^{3}$ Licenciado en Ciencias Económicas, Máster en Informática - Universidade Pinar del Rio - Rúa C, 111 - 20.100-000 - Pinar del Río, Cuba jluis@infomed.sld.cu
}

Cerne, Lavras, v. 18, n. 1, p. 33-40, jan./mar. 2012 
rigurosa de todas las operaciones de la cosecha de madera (AMARAL et al., 1998; BECKER, 1994; HENDRISON, 1990; KLUENDER; STOKES, 1996; MALINOVSKI; MALINOVSKI, 2000).

La evaluación detallada de los sistemas de aprovechamiento de madera ha mostrado que las operaciones arrastre, transporte y construcción de camino tienen generalmente la mayor influencia en el costo total del sistema (AMARAL et al., 1998; KLUENDER; STOKES, 1996; LANFORD; STOKES, 1995). También las operaciones de arrastre de madera y construcción de caminos tienen gran repercusión en los daños provocados a los ecosistemas forestales durante el aprovechamiento (AMARAL et al., 1998; HENDRISON, 1990; KILLMANN et al., 2002; WINKLER, 1997).

El cálculo del tamaño del equipo de trabajo apropiado para cada máquina, el punto de equilibrio en una operación, la determinación de la densidad óptima de caminos y patios de carga y la calidad de la capa de rodamiento de los caminos en base a obtener un costo mínimo, han sido estudiado por varios autores pero de forma separada (DAGANG et al., 2002; DYKSTRA; HEINRICH, 1997; MACHADO et al., 1991; SILVA et al., 1991; WINKLER, 1997).

Teniendo en consideración lo expuesto, se realiza este trabajo con el objetivo de minimizar el costo del sistema de aprovechamiento de madera en bosques de Pinus caribaea, a partir de la interacción entre el costo de camino y del arrastre de madera en base a la densidad de camino y patios de carga, además de la interacción del costo de camino y de transporte de madera en base a la calidad de la capa de rodamiento del camino.

\section{MATERIALES Y METODO}

\section{1 Área de estudio}

El experimento se realizó en la empresa forestal $\mathrm{La}$ Palma en la provincia de Pinar del Río, en el occidente de la isla de Cuba, localizada en los $\left(83^{\circ} 50^{\prime} \mathrm{N}\right.$ y $22^{\circ} 55^{\prime}$ W), a una altitud de $127 \mathrm{msnm}$. El estudio se desarrolló en rodales naturales de Pinus caribaea, sometidos a la tala rasa, donde el diámetro promedio de los árboles fue de $24,6 \mathrm{~cm}$, la altura de $15,3 \mathrm{~m}$ y el volumen promedio de los árboles de $0,31 \mathrm{~m}^{3}$, el volumen total de $125 \mathrm{~m}^{3} /$ ha. La temperatura media anual del sitio es de $23,8^{\circ}$, la humedad relativa de $74 \%$, la precipitación anual promedio de 1.486,6 $\mathrm{mm}$. El terreno tiene un relieve ondulado, con pendiente entre 12 y $28 \%$. El suelo se clasifica como Loam arcilloso arenoso.

Cerne, Lavras, v. 18, n. 1, p. 33-40, jan./mar. 2012

\subsection{Metodología}

Se calculó el costo del sistema de aprovechamiento de madera utilizado en la empresa forestal la Palma. Los estudios de tiempo y productividad se determinaron según los criterios de Rickards et al. (1995). Con el auxilio del sistema automatizado PACE (SESSIONS, 1992), se calculó el costo de las operaciones.

Para el cálculo del costo por unidad de producción, referente a caminos y patios de carga se uso la siguiente expresión:

$$
\mathrm{Cuc}=\frac{\mathrm{Cr} *\left(\mathrm{~L} / 10^{3}\right)+\mathrm{Cl}}{\mathrm{V}^{*} \mathrm{~S} * \mathrm{~L} / 10^{4}}
$$

donde:

Cuc - Costo unitario de caminos y patios de carga, $\left(\$ / \mathrm{m}^{3}\right)$.

$\mathrm{Cr}-$ Costo de construcción del camino, $(\$ / \mathrm{km})$.

$\mathrm{V}$ - Volumen de madera utilizada por unidad de área, $\left(\mathrm{m}^{3} / \mathrm{ha}\right)$.

$\mathrm{Cl}$ - Costo de construcción de patio, (\$).

$\mathrm{L}$ - Espaciamiento promedio entre patios $(\mathrm{m})$.

$\mathrm{S}$ - Espaciamiento promedio entre caminos (m).

Para el cálculo del costo de arrastre de madera se utilizó la expresión:

$$
\text { Cua }=\frac{\text { Cef }+ \text { Ceop }+ \text { Celb }}{\frac{\mathrm{V}^{*}[60-\mathrm{Ti}]}{\operatorname{da}}+\mathrm{T} a+\frac{d a}{\operatorname{VrcC}}+\mathrm{Td}}
$$

donde:

Cua - Costo unitario de arrastre de madera, $\left(\$ / \mathrm{m}^{3}\right)$.

Cef - Costo fijo del tractor, $(\$ / h)$.

Ceop - Costo variable del tractor, $(\$ / \mathrm{h})$.

Celb - Costo de mano de obra, $(\$ / \mathrm{h})$.

$\mathrm{V}$ - Volumen arrastrado o carga útil del tractor, $\left(\mathrm{m}^{3}\right)$.

$\mathrm{Ti}$ - Tiempo de interrupciones, $(\mathrm{min} / \mathrm{h})$.

$d a$ - Distancia promedio de arrastre, (m).

Vrsc - Velocidad sin carga del tractor, $(\mathrm{m} / \mathrm{min}$.).

Vrcc - Velocidad con carga del tractor, $(\mathrm{m} / \mathrm{min}$.).

Ta - Tiempo de amarre de la madera, (min.).

$\mathrm{T} d$ - Tiempo de desamarre, (min.).

Para el costo del transporte fue empleada una expresión muy semejante:

$$
\mathrm{Cua}=\frac{\text { Cef }+ \text { Ceop }+ \text { Celb }}{\frac{\mathrm{V}}{V t s c}+\mathrm{Tc}+\frac{d t}{V r c c}+\mathrm{Td}}
$$


donde:

Cut - Costo unitario del transporte de madera, $\left(\$ / \mathrm{m}^{3}\right)$.

Cef - Costo fijo del camión, $(\$ / h)$.

Ceop - Costo variable del camión, $(\$ / h)$.

Celb - Costo de mano de obra, $(\$ / \mathrm{h})$.

$\mathrm{V}$ - Volumen de carga del camión, $\left(\mathrm{m}^{3}\right)$.

$d t$ - Distancia promedio de transporte, $(\mathrm{m})$.

Vrsc - Velocidad sin carga del camión, $(\mathrm{km} / \mathrm{h})$.

Vrcc - Velocidad con carga del camión, $(\mathrm{km} / \mathrm{h})$.

Tc - Tiempo de carga del camión, (h).

Td - Tiempo de descarga, (h).

\subsection{Aplicación de criterios para minimizar el costo del sistema de aprovechamiento}

Se aplicaron dos criterios para optimizar el costo del sistema de aprovechamiento (Figura 1). El primero, la interacción del costo de construcción de camino y patio $\mathrm{y}$ el costo de arrastre de madera en base a una densidad de caminos donde la sumatoria del costo de construcción de caminos y el costo de arrastre se minimiza.

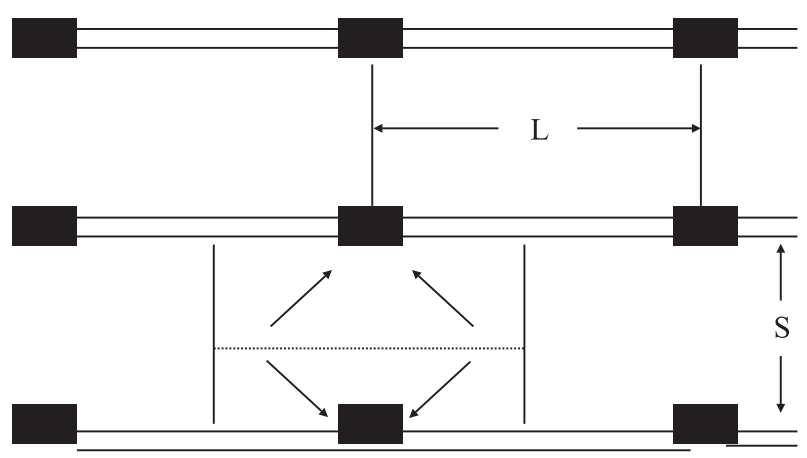

S - Espaciamiento entre caminos.

$\mathrm{L}$ - Espaciamiento entre patios.

$\mathrm{da}=f(\mathrm{~S}, \mathrm{~L})$

Figura 1 - Esquema del espaciamiento entre caminos y entre patios de carga.

Figure 1 - Scheme of the spacing among roads and among loading patios.

Como la distancia de arrastre (da), es una función del espaciamiento promedio entre caminos y entre patios e interviene en el costo de construcción de caminos y también en el costo del arrastre, se utilizo la expresión que sirve de interacción entre ambas operaciones:

$$
\begin{aligned}
\mathrm{da}= & \left\{1 / 3 *\left[(0,5 * \mathrm{~S})^{2}+(\mathrm{L})^{2}\right)\right]^{0,5}+1 / 3 *\left[(0,25 * \mathrm{~S})^{2}+\right. \\
& \left.\left.\left.(0,5 * \mathrm{~L})^{2}\right)\right]^{0,5}\right\}^{*} \mathrm{k}
\end{aligned}
$$

donde:

$\mathrm{S}$ - Espaciamiento promedio entre caminos, (m).

$\mathrm{L}$ - Espaciamiento promedio entre patios, $(\mathrm{m})$.

$\mathrm{k}$ - Coeficiente de sinuosidad, $(\mathrm{k} \geq 1)$. Relación entre la distancia real de arrastre y la distancia teórica.

La distancia promedio de arrastre calculada en la expresión (4), contiene el espaciamiento promedio entre caminos (S) y el espaciamiento promedio entre patios (L). Entonces, el costo de camino y el costo de arrastre estarán determinados por estas dos variables. Como son muchas las posibles interacciones, se utilizó el Software PACE, que permite automatizar estos cálculos y determinar el espaciamiento promedio óptimo entre caminos y entre patios de carga y a su vez una distancia de arrastre que minimiza el costo de ambas operaciones.

El segundo criterio para minimizar el costo del sistema de aprovechamiento, fue la interacción entre el costo de construcción de camino y el costo del transporte. El trabajo evalúa el costo de las actividades de construcción de camino $(\mathrm{Cr})$ referido en la expresión (1), primero para el método tradicional de construcción de $6,5 \mathrm{~m}$ de ancho de calzada y después, con la reducción del ancho de la calzada a 4,5 $\mathrm{m}$ y el aumento de la calidad de la capa de rodamiento del camino, que permitiera mayores velocidades de transporte.

$\mathrm{Cr}=\Sigma$ (Costo de las operaciones de construcción del camino)

En esta interacción se modifica, el costo de construcción del camino $(\mathrm{Cr})$ al utilizar un ancho de calzada menor y un mejoramiento en la operación del perfilado de la superficie de este. A consecuencia, la velocidad de recorrido de los camiones de transporte contenida en la expresión (3) son influenciadas y repercute por tanto en el costo del transporte. Se calculó el costo unitario de camino para ambas opciones, versus costo unitario del transporte.

\subsection{Composición del sistema de aprovechamiento}

Todos los operadores y ayudantes que trabajaron con las máquinas que realizaron las operaciones tenían más de dos años de experiencia (Tabla 1). Fueron evaluadas 15 jornadas de trabajo completa en cada operación, para tomar la información necesaria de tiempos utilizados en cada operación y consumo de combustibles, lubricantes y otros materiales que consume la maquinaria, además del inventario del salario y los beneficios de cada trabajador.

Cerne, Lavras, v. 18, n. 1, p. 33-40, jan./mar. 2012 
Tabla 1 - Maquinaria y el numero de trabajadores empleados en el sistema de aprovechamiento de madera evaluado.

Table 1 - Machinery and number of workers employed in timber harvesting system evaluated.

\begin{tabular}{|c|c|}
\hline Operación & Maquinaria \\
\hline Tala y desrame & $\begin{array}{l}\text { Motosierra STIHL }(87 \mathrm{cc}) \text { con } 1 \text { operador } \\
+1 \text { ayudante }\end{array}$ \\
\hline Arrastre & $\begin{array}{l}\text { Tractor TDT- } 55 \text { A, de orugas con } 1 \\
\text { operador }+2 \text { ayudantes }\end{array}$ \\
\hline Carga & $\begin{array}{l}\text { Gargador Frontal PL-2, de orugas o esteras } \\
\text { con } 1 \text { operador }\end{array}$ \\
\hline Transporte & Camión semirremolque MAZ-509, 1 operador \\
\hline $\begin{array}{l}\text { Construcción } \\
\text { caminos y patios }\end{array}$ & $\begin{array}{l}\text { Bulldozer marca KOMATZU- D85A, } 1 \\
\text { operador. }\end{array}$ \\
\hline
\end{tabular}

Se utilizaron los modelos estadísticos desarrollados por (CÁNDANO, 1998) para estimar el tiempo de ejecución, la productividad y el costo de cada operación. El paquete estadístico SPSS, fue utilizado para procesar, obtener los modelos de regresión y realizar el ajuste de los mismos. Siempre se trabajo para error máximo admisible del $10 \%$ y una probabilidad del $95 \%$.

\section{RESULTADOS Y DISCUSION}

3.1 Costos del sistema de aprovechamiento en función del espaciamiento óptimo entre caminos y patios de carga

En la Tabla 2, se muestra la gran repercusión de los costos de arrastre, transporte y construcción de caminos en el costo total del sistema de aprovechamiento de madera. Otra tendencia en sentido general de gran importancia para las empresas, es que más del 50\% de los costos del sistema de aprovechamiento pertenecen al costo de operación y dentro de este, el costo de combustible y lubricante tienen la mayor incidencia.
La baja productividad de las operaciones de corte y arrastre está asociada con la pequeña dimensión de los árboles en los rodales naturales de Pinus caribaea y a su vez, el bajo volumen de madera por unidad de superficie.

\subsection{Resultados de la aplicación del criterio de la densidad óptima de caminos y patios de carga}

Primeramente, se determinó en el área de trabajo la distancia promedio entre caminos (S) que fue de 410 metros y la distancia promedio entre patios (L) de 106 metros. Además de determinar el coeficiente de sinuosidad (k) que resulto 1,20. Para estos valores se aplicó la expresión (4) y el resultado de la distancia promedio de arrastre fue:

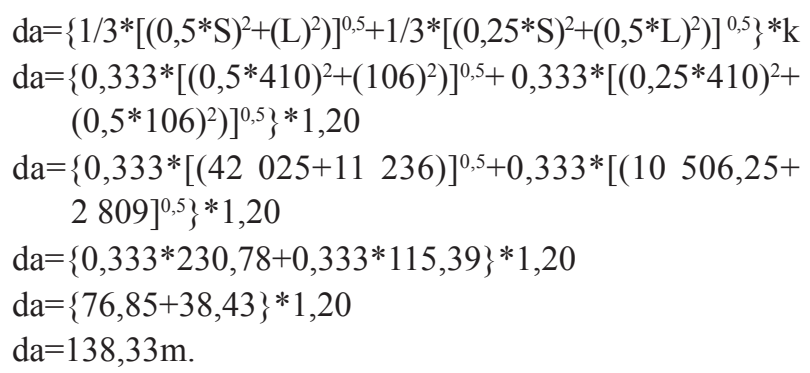

Con este valor de la distancia de arrastre y los valores de (S) y (L) utilizados para el cálculo de la distancia de arrastre se determinó el costo de ambas operaciones sustituyendo en de las expresiones (1) y (2).

$$
\begin{aligned}
\mathrm{Cua} & =\frac{10,36+10,18+1,26}{2,24 *[60-9]} \\
\mathrm{Cua}= & 21,8 /(91,8 /(1,61+6,11+2,03+2,07)) \\
\mathrm{Cua}= & 21,8 /(114,53 / 11,82) \\
\mathrm{Cua}= & 21,8 / 9,69 \\
\mathrm{Cua}= & 2,25 \$ / \mathrm{m}^{3} .
\end{aligned}
$$

Tabla 2 - Resumen del costo del sistema de aprovechamiento.

Table 2 - Summary of the cost of the wood use system.

\begin{tabular}{lccccc}
\hline Operaciones & $\begin{array}{c}\text { Productividad } \\
\left(\mathrm{m}^{3} / \mathrm{h}\right)\end{array}$ & $\begin{array}{c}\text { Costo fijo } \\
\left(\$ / \mathrm{m}^{3}\right)\end{array}$ & $\begin{array}{c}\text { Costo de operación } \\
\left(\$ / \mathrm{m}^{3}\right)\end{array}$ & $\begin{array}{c}\text { Costo de mano } \\
\text { de obra }\left(\$ / \mathrm{m}^{3}\right)\end{array}$ & $\begin{array}{c}\text { Costo total } \\
\left(\$ / \mathrm{m}^{3}\right)\end{array}$ \\
\hline Corte & 4,39 & 0,11 & 0,28 & 0,37 & 0,76 \\
Arrastre & 9,69 & 1,07 & 1,06 & 0,13 & 2,25 \\
Carga & 41,67 & 0,38 & 0,31 & 0,03 & 0,72 \\
Transporte & 6,44 & 1,09 & 2,94 & 0,18 & 4,21 \\
Caminos & & 0,96 & 0,96 & 0,14 & 2,06 \\
\hline Total & & 3,60 & 5,55 & 0,85 & 10,00 \\
\hline
\end{tabular}

1 Peso convertible cubano (CUC) $=1$ Dólar estadounidense (USD).

Cerne, Lavras, v. 18, n. 1, p. 33-40, jan./mar. 2012 


$$
\begin{aligned}
& \mathrm{Cuc}=\frac{9858,36 *\left(106 / 10^{3}\right)+75,96}{125 * 410^{*} 106 / 10^{4}} \\
& \mathrm{Cuc}=(9858,36 * 0,106+75,96) / 543,25 \\
& \mathrm{Cuc}=1044,99 * 75,96 / 195,57 \\
& \mathrm{Cuc}=1 \quad 120,95 / 195,57 \\
& \mathrm{Cuc}=2,06 \$ \mathrm{~m}^{3} .
\end{aligned}
$$

Se obtiene un costo total de $4,31 \$ / \mathrm{m}^{3}$ para ambas operaciones. Después de la interacción automatizada se obtuvo que el espaciamiento promedio entre caminos y entre patios que minimiza el costo total es 721,12 y 123,01 $\mathrm{m}$ respectivamente.

Sustituyendo estos valores se obtiene una distancia promedio de arrastre de:

$$
\begin{aligned}
\mathrm{da}= & \left.\left.\left.1 / 3 *\left[\left(0,5^{*} \mathrm{~S}\right)^{2}+(\mathrm{L})^{2}\right)\right]^{0,5}+1 / 3 *\left[(0,25 * \mathrm{~S})^{2}+(0,5 * \mathrm{~L})^{2}\right)\right]^{0,5}\right\} * \mathrm{k} \\
\mathrm{da}= & \left\{0,333^{*}\left[\left(0,5^{*} 721,12\right)^{2}+(123,01)^{2}\right)\right]^{0,5}+0,333^{*}[(0,25 * \\
& \left.\left.\left.721,12)^{2}+\left(0,5^{*} 123,01\right)^{2}\right)\right]^{0,5}\right\} * 1,20 \\
\mathrm{da}= & \left\{0,333 *[(130003,51+15131,46)]^{0,5}+0,333^{*}[(32.500,89+\right. \\
& \left.3.782,87]^{0,5}\right\}^{*} 1,20 \\
\mathrm{da}= & \{0,333 * 380,97+0,333 * 190,48\} * 1,20 \\
\mathrm{da}= & \{126,86+63,43\} * 1,20 \\
\mathrm{da}= & 228,35 \mathrm{~m} .
\end{aligned}
$$

Con el nuevo valor de la distancia de arrastre obtenido y los valores de (S) y (L), se determina el costo de ambas operaciones.

$$
\mathrm{Cua}=\frac{10,36+10,18+1,26}{\frac{2,24 *[60-9]}{\frac{228,35}{86}+6,11+\frac{228,35}{68}+2,07}}
$$

Cua $=2,70 \$ / \mathrm{m}^{3}$

$$
\begin{aligned}
& \mathrm{Cuc}=\frac{9858,36 *\left(123,01 / 10^{3}\right)+75,96}{125 * 721,12 * 123,01 / 10^{4}} \\
& \mathrm{Cuc}=1,16 \$ \mathrm{~m}^{3}
\end{aligned}
$$

Utilizando los valores propuestos, se observa un aumento de 90,17 $\mathrm{m}$ en la distancia de arrastre, disminuyendo la productividad del tractor en $1,65 \mathrm{~m}^{3} / \mathrm{h}$ efectiva y aumentando el costo del arrastre en $0,45 \$ / \mathrm{m}^{3}$, simultáneamente, la densidad de camino de $24,39 \mathrm{~m} / \mathrm{ha}$ disminuye para $13,87 \mathrm{~m} /$ ha y aunque el espaciamiento de patios aumenta poco, el costo de construcción de caminos y patios se reduce en $0,90 \$ / \mathrm{m}^{3}$.

Como resultado del efecto de la interacción se logra disminuir el costo total por la aplicación de este criterio en $0,45 \$ / \mathrm{m}^{3}$ y el impacto ambiental provocado por la construcción de caminos disminuye aproximadamente un $44 \%$.

También es muy importante la interacción entre la calidad o características constructivas del camino y los indicadores de eficiencia del transporte. Casi siempre en el aprovechamiento hay que planificar en base a un presupuesto bien limitado y utilizar los recursos de la forma más efectiva posible.

\subsection{Análisis del costo del sistema de aprovechamiento en función de la calidad de la capa de rodamiento de los caminos de transporte}

El análisis se ha realizado manteniendo el costo aproximado de camino que garantice aumentar la velocidad de transporte (Tabla 3). Es importante destacar que se ha conservado el mismo espaciamiento de camino, para no afectar el costo del arrastre y a su vez, del sistema de aprovechamiento.

Tabla 3 - Resumen del costo del sistema de aprovechamiento en base a densidad óptima.

Table 3 - Summary of the cost of the system based on optimal density.

\begin{tabular}{lccccc}
\hline Operaciones & $\begin{array}{c}\text { Productividad } \\
\left(\mathrm{m}^{3} / \mathrm{h}\right)\end{array}$ & $\begin{array}{c}\text { Costo fijo } \\
\left(\$ / \mathrm{m}^{3}\right)\end{array}$ & $\begin{array}{c}\text { Costo de operación } \\
\left(\$ / \mathrm{m}^{3}\right)\end{array}$ & $\begin{array}{c}\text { Costo de mano } \\
\text { de obra }\left(\$ / \mathrm{m}^{3}\right)\end{array}$ & $\begin{array}{c}\text { Costo total } \\
\left(\$ / \mathrm{m}^{3}\right)\end{array}$ \\
\hline Corte & 4,39 & 0,11 & 0,28 & 0,37 & 0,76 \\
Arrastre & 9,69 & 1,07 & 1,06 & 0,13 & 2,70 \\
Carga & 41,67 & 0,38 & 0,31 & 0,03 & 0,72 \\
Transporte & 6,44 & 1,09 & 2,94 & 0,18 & 4,21 \\
Caminos & & 0,96 & 0,96 & 0,14 & 1,16 \\
\hline Total & & 3,60 & 5,55 & 0,85 & 9,55 \\
\hline
\end{tabular}

1 Peso convertible cubano (CUC) = 1 Dólar estadounidense (USD).

Cerne, Lavras, v. 18, n. 1, p. 33-40, jan./mar. 2012 


$$
\begin{aligned}
& \text { Cut }=\frac{\text { Cef }+ \text { Ceop }+ \text { Celb }}{\frac{\mathrm{V}}{\operatorname{lt}}+\mathrm{Tc}+\frac{d t}{\operatorname{Vrcc}}+\mathrm{Td}} \\
& \mathrm{Cut}=\frac{27,11}{\frac{14}{\frac{23}{30}+0,32+\frac{23}{25}+0,17}} \\
& \mathrm{Cut}=\frac{27,11}{6,44} \\
& \mathrm{Cut}=4,21 \$ / \mathrm{m}^{3}
\end{aligned}
$$

En la condición del camino con capa de rodamiento sin perfilar adecuadamente para el transporte con una calzada de $6,5 \mathrm{~m}$ de ancho la productividad del transporte de madera fue de $6,44 \mathrm{~m}^{3} /$ hora efectiva de trabajo. La velocidad promedio de recorrido del camión es de 27,5 $\mathrm{km} / \mathrm{h}$, muy baja con respecto a resultados internacionales.

Para estos parámetros tradicionales el costo del camino es de 9.858,00 \$/km construido en condiciones de suelo con pendiente y baja aptitud para la construcción.

Como se puede apreciar en la Tabla 4, lo más significativo es el alto costo de la operación de terraplén (corte de tierra y relleno). También hay que destacar en sentido general la repercusión del costo fijo y del costo variable dentro del costo total de construcción del camino.

Debe señalarse que los operadores de bulldozer producen muchos cortes y movimientos de tierra innecesarios, lo que provoca gastos de recursos como combustibles y lubricantes y horas máquinas de trabajo.

Después de colocar en práctica parámetros diferentes para la construcción del camino, como el ancho de calzada de 4,5 m, con ensanchamientos necesarios en las curvas y el mejoramiento en el perfilado de la capa de rodamiento, se aprecia una disminución notoria en el costo del transporte.

$$
\text { Cut }=\frac{\text { Cef }+ \text { Ceop }+ \text { Celb }}{\frac{\mathrm{V}}{\operatorname{Vrsc}}+\mathrm{Tc}+\frac{d t}{V r c c}+\mathrm{Td}}
$$

$$
\text { Cut }=\frac{27,11}{\frac{14}{\frac{23}{45,02}+0,32+\frac{23}{35,17}+0,17}}
$$

Cut $=\frac{27,11}{8,48}$

Cut $=3,20 \$ / \mathrm{m}^{3}$

Al mejorar la capa de rodamiento se produce un aumento en la velocidad promedio de recorrido del camión, aumenta la productividad del transporte en $2,04 \mathrm{~m}^{3} /$ hora efectiva de trabajo y disminuye aproximadamente el 24 $\%$ del costo unitario.

Al analizar las actividades en la construcción de camino en la Tabla 5, se puede notar que hay poca diferencia del costo total con respecto a la Tabla 4, pero se disminuye el costo de terraplén significativamente. Esto se explica porque se redujo el ancho de la calzada del camino, disminuyendo el volumen de trabajo de esta actividad y simultáneamente hay un aumento en el costo de perfilado del $50 \%$ aproximadamente, dado por el mayor volumen trabajo de esta operación en la capa de rodamiento.

La aplicación del criterio relacionado con el ancho de la calzada, la capa de rodamiento y el transporte, reduce el costo del sistema de aprovechamiento en 1,01 \$/ $\mathrm{m}^{3}$ (Tabla 6). En este caso específico tiene mayor repercusión económica que la densidad de caminos y patios.

Tabla 4 - Resumen del costo de construcción de caminos por método tradicional.

Table 4 - Summary of the cost of road construction according to the traditional method.

\begin{tabular}{lcccc}
\hline Actividades & Costo fijo $(\$ / \mathrm{km})$ & Costo de operación $(\$ / \mathrm{km})$ & Costo de mano de obra $(\$ / \mathrm{km})$ & Costo total $(\$ / \mathrm{km})$ \\
\hline Trazado & 0,00 & 0,00 & 221,00 & 221,00 \\
Desmonte y limpieza & 37,00 & 113,00 & 82,00 & 232,00 \\
Terraplén & $3.349,00$ & $3.154,00$ & 165,00 & $6.669,00$ \\
Formación & 343,00 & 387,00 & 29,40 & 759,00 \\
Drenaje & 498,00 & 438,00 & 18,00 & 954,00 \\
Perfilado & 369,00 & 474,00 & 180,00 & $1.023,00$ \\
\hline Totales & $4.597,00$ & $4.566,00$ & 695,00 & $9.858,00$ \\
\hline
\end{tabular}

1 Peso convertible cubano (CUC) = 1 Dólar estadounidense (USD).

Cerne, Lavras, v. 18, n. 1, p. 33-40, jan./mar. 2012 
Tabla 5 - Resumen del costo de construcción de camino por método propuesto.

Table 5 - Summary of road construction costs for the proposed method.

\begin{tabular}{lrcrc}
\hline Actividades & $\begin{array}{c}\text { Costo fijo } \\
(\$ / \mathrm{km})\end{array}$ & $\begin{array}{c}\text { Costo de operación } \\
(\$ / \mathrm{km})\end{array}$ & $\begin{array}{c}\text { Costo de mano } \\
\text { de obra }(\$ / \mathrm{km})\end{array}$ & $\begin{array}{c}\text { Costo total } \\
(\$ / \mathrm{km})\end{array}$ \\
\hline Trazado & 0,00 & 0,00 & 221,00 & 221,00 \\
Desmonte y limpieza & 31,00 & 94,00 & 68,00 & 193,00 \\
Terraplén & $2.093,00$ & $2.023,00$ & 113,00 & $4.230,00$ \\
Formación & 429,00 & 483,00 & 37,00 & 949,00 \\
Drenaje & 498,00 & 438,00 & 18,00 & 954,00 \\
Perfilado & $1.307,00$ & $1.491,00$ & 471,00 & $3.269,00$ \\
\hline Totales & $4.358,00$ & $4.530,00$ & 928,00 & $9.816,00$ \\
\hline
\end{tabular}

1 Peso convertible cubano (CUC) = 1 Dólar estadounidense (USD).

Tabla 6 - Resumen del costo del sistema de aprovechamiento minimizado.

Table 6 - Summary of the cost of the system of the minimal use.

\begin{tabular}{lccccc}
\hline Operaciones & $\begin{array}{c}\text { Productividad } \\
\left(\mathrm{m}^{3} / \mathrm{h}\right)\end{array}$ & $\begin{array}{c}\text { Costo fijo } \\
\left(\$ / \mathrm{m}^{3}\right)\end{array}$ & $\begin{array}{c}\text { Costo de operación } \\
\left(\$ / \mathrm{m}^{3}\right)\end{array}$ & $\begin{array}{c}\text { Costo de mano } \\
\text { de obra }\left(\$ / \mathrm{m}^{3}\right)\end{array}$ & $\begin{array}{c}\text { Costo total } \\
\left(\$ / \mathrm{m}^{3}\right)\end{array}$ \\
\hline Corte & 4,39 & 0,11 & 0,28 & 0,37 & 0,76 \\
Arrastre & 9,69 & 1,07 & 1,06 & 0,13 & 2,70 \\
Carga & 41,67 & 0,38 & 0,31 & 0,03 & 0,72 \\
Transporte & 8,48 & 1,09 & 1,93 & 0,18 & 3,20 \\
Caminos & & 0,96 & 0,96 & 0,14 & 1,16 \\
\hline Total & & 5,60 & 5,55 & 0,85 & 8,54 \\
\hline
\end{tabular}

1 Peso convertible cubano (CUC) = 1 Dólar estadounidense (USD).

\section{CONCLUSIONES}

La densidad de caminos que minimiza el costo del sistema de aprovechamiento en rodales naturales de Pinus caribaea en Pinar del Rio-Cuba, es 13,87 m/ha, o sea espaciamiento de $721 \mathrm{~m}$ y $123 \mathrm{~m}$ entre patios de carga respectivamente.

La construcción de caminos de transporte con una reducción del ancho de la calzada de 6,5 m a 4,5 m y un mejoramiento en el perfilado del camino aumenta la velocidad del camión, la productividad del transporte y reduce el costo del sistema de cosecha de madera.

La aplicación de ambos criterios, produce un efecto económico del 14, $60 \%$ sobre el costo total del sistema de cosecha, con $4,5 \%$ referido a la densidad de caminos y patios y el 10,1\% a las características constructivas del camino y su relación con el transporte.

El procedimiento mostrado en este trabajo puede ser generalizado para minimizar el costo de cualquier sistema de cosecha de madera, desde que se obtengan las informaciones necesarias y precisas para el análisis, con independencia del tipo de maquinaria y características del bosque.
Cualquier análisis de costo sobre sistemas de cosecha de madera, es necesario realizarlo de forma holística, por la interacción que una operación puede tener sobre el costo de otras.

Es posible minimizar el costo de un sistema de cosecha de madera y simultáneamente reducir los impactos ambientales derivados de la construcción excesiva de caminos y patios de carga.

\section{REFERENCIAS}

AMARAL, P.; VERISSIMO, A.; BARRETO, P. E. Floresta

para sempre: um manual para a produção de madeira na Amazônia. Belém: UFPA, 1998. 155 p.

BECKER, G. Optimization of road network and transport systems: a pré-condition for an improved organization and design of labour in forestry. In: SEMINÁRIO DE ATUALIZAÇÃO SOBRE SISTEMAS DE COLHEITA DE MADEIRA E TRANSPORTE FLORESTAL, 8., 1994, Curitiba. Anais... Curitiba: FUPEF, 1994. p. 111-115.

Cerne, Lavras, v. 18, n. 1, p. 33-40, jan./mar. 2012 
CÁNDANO, F. Propuesta para incrementar la eficiencia del sistema de aprovechamiento de madera en rodales de Pinus caribaea en la provincia de Pinar del Río-Cuba. 1998. 141 p. Tesis (Doctorado en Ciencias Forestales) Universidad de Pinar del Río, Pinar del Río, 1998.

DAGANG, A. A.; RICHTER, F.; HAHN-SCHILLING, B.; MANGGIL, P. Financial and economic analyses of conventional and reduced impact harvesting systems in Sarawak. In: _. Applying reduced impact logging to advance sustainable forest management. Bangkok: AsiaPacific Forestry Commission, 2002. p. 151-162.

DYKSTRA, D. P.; HEINRICH, R. Forest harvesting and transport: old problems, new solutions. In: WORLD FORESTRY CONGRESS, 3., 1997, Antalya. Proceedings... Antalya, 1997. p. 171-186.

HENDRISON, J. Damage-controlled logging in managed tropical rainforest in Suriname. Wageningen: Agricultura University, 1990. 199 p.

KILLMANN, W.; BULL, G. Q.; SCHWAB, O.; PULKKI, R. E. Reduced impact logging: does it cost or does it pay?: applying reduced impact logging to advance sustainable forest management. Bangkok: Asia-Pacific Forestry Commission, 2002. p. 107-124.

KLUENDER, R. A.; STOKES, B. J. Felling and skidding productivity and harvesting cost in southern pine forests. In: JOINT CONFERENCE CANADIAN WOODLANDS FORUM, 1996, Quebec. Proceedings... Quebec: CPPA/ IUFRO, 1996. p. 5.

LANFORD, B. L.; STOKES, B. J. Cost and productivity comparison of two thinning systems. Washington: American Palpwood Association, 1995. 12 p.

MACHADO, C. C.; SANTOS, S. L. M.; FONTES, J. M. Influência da qualidade da rodovia no custo de transporte florestal. In: SIMPÓSIO BRASILEIRO SOBRE EXPLORAÇÃO E TRANSPORTE FLORESTAL, 1., 1991, Belo Horizonte. Anais... Viçosa, MG: UFV, 1991. p. 1-18.
MALINOVSKI, R. A.; MALINOVSKI, J. R. Programa de computação para simulação e controle de operações de colheita de madeira. In: SEMINÁRIO DE ATUALIZAÇÃO SOBRE SISTEMAS DE COLHEITA DE MADEIRA E TRANSPORTE FLORESTAL, 11., 2000, Curitiba. Anais... Curitiba: UFPR, 2000. p. 10.

PETERS, P. A. Spacing of roads and landings to minimize timber harvest cost. Forestry Science, Amsterdam, v. 24, n. 2, p. 209-217, 1978.

RICKARDS, J.; SKAAR, R.; HABERLE, S.; APEL, K.; BJORHEDEN, R.; THONPSOM, M. Forest work study nomenclature. In: CONGRESS IUFRO, 5., 1995, Tampere. Proceedings... Tampere, 1995. p. 16.

SESSIONS, J. Cost control in forest harvesting and road construction. Rome: FAO, 1992. 106 p. (Forestry paper, 99).

SESSIONS, J.; GUANGDA, L. Deriving optimal road and landing with microcomputer programs. Western Journal of Applied Forestry, Madison, v. 2, n. 3, p. 94-98, July 1987.

SESSIONS, J.; HUAT, Y. Optimizing road spacing and equipment allocation simultaneously. Forest Products Journal, Corvallis, v. 39, n. 10, p. 43-46, 1989.

SILVA, C. E.; MACHADO, C. C.; SOUZA, A. P. Otimização da produtividade e do custo da extração florestal com guincho arrastador. In: SIMPÓSIO BRASILEIRO SOBRE EXPLORAÇÃO E TRANSPORTE FLORESTAL, 1., 1991, Belo Horizonte. Anais... Viçosa, MG: UFV, 1991. p. 113-126.

SUDDARTH, S. K.; HEINRICH, A. M. Average skidding distance for theoretical analysis of logging costs. Indiana: Pardue University Agriculture Exposition Station Lafayette, 1964. $6 \mathrm{p}$.

WINKLER, N. Aprovechamiento forestal compatible con el medio ambiente: ensayo sobre la aplicación del Código Modelo de la FAO en la Amazona Brasileña. Rome: FAO, 1997. 84 p. (Estudio monográfico, 8).

Cerne, Lavras, v. 18, n. 1, p. 33-40, jan./mar. 2012 